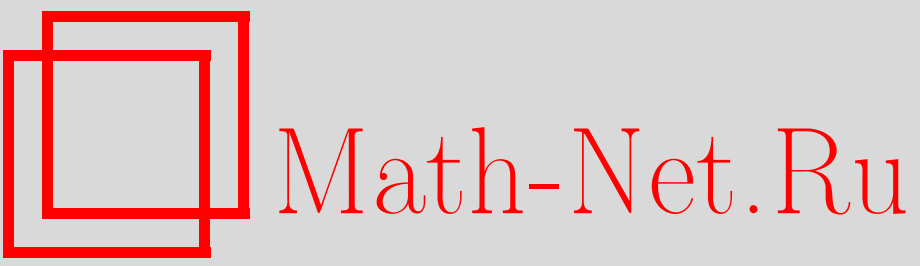

Г. Фикера, Об одной граничной задаче, связанной с реакцией полупространства на короткий лазерный пульс, УМН, 1997, том 52, выпуск 2, 191-192

DOI: https://doi.org/10.4213/rm846

Использование Общероссийского математического портала Math-Net.Ru подразумевает, что вы прочитали и согласны с пользовательским соглашением

http://www.mathnet.ru/rus/agreement

Параметры загрузки:

IP: 3.85 .73 .92

26 апреля 2023 г., 04:42:15 


\title{
ОБ ОДНОЙ ГРАНИЧНОЙ ЗАДАЧЕ, СВЯЗАННОЙ С РЕАКЦИЕЙ ПОЛУПРОСТРАНСТВА НА КОРОТКИЙ ЛАЗЕРНЫЙ ПУЛЬС
}

\author{
Г. ФИКЕРА
}

Задача, которая изучается в этой заметке, была рассмотрена Р. Хетнарским и Ж. Игнацаком [1] В рамках обобщенной термоупругости. Из анализа задачи они вывели важные механические следствия. Здесь приводится математическое исследование этой задачи. Она сводится к рассмотрению следующей смешанной граничной задачи

$$
\begin{gathered}
\Gamma \varphi=f(x, t) \quad \text { в } Q, \quad \frac{\partial^{k} \varphi}{\partial t^{k}}(x, 0)=0, \quad k=0,1,2,3, \quad x>0, \\
\frac{\partial^{k} \varphi}{\partial x^{k}}(0, t)=0, \quad k=0,2, \quad t>0,
\end{gathered}
$$

где $\Gamma \equiv \frac{\partial^{4}}{\partial x^{4}}+t_{0} \frac{\partial^{4}}{\partial t^{4}}-\left(1+t_{0}+\varepsilon t^{0}\right) \frac{\partial^{4}}{\partial x^{2} \partial t^{2}}+\frac{\partial^{3}}{\partial t^{3}}-(1+\varepsilon) \frac{\partial^{3}}{\partial x^{2} \partial t}, Q=\{x, t: x>0, t>0\}$, $t_{0}, t^{0}, \varepsilon$ - некоторые положительные постоянные.

Предполагается, что выполнено условие $H_{0}$ : функции $f, \frac{\partial f}{\partial x}, \frac{\partial f}{\partial t}, \frac{\partial^{2} f}{\partial x \partial t}$ непрерьвны в $\bar{Q}$. Решение задачи (1) ищется в классе $C^{3}(\bar{Q}) \cap H^{4}(E)$, где $E$ - любая ограниченная область, содержащаяся в $Q$. Для решения задачи (1) рассматривается вспомогательная задача

$$
\begin{gathered}
\Gamma u=f(x, t) \quad \text { в } \quad R_{a, T}, \quad u(0, t)=\frac{\partial^{2} u(0, t)}{\partial x^{2}}=u(a, t)=\frac{\partial^{2} u(a, t)}{\partial x^{2}}=0, \quad 0<t<T, \\
\frac{\partial^{k} u(x, 0)}{\partial t^{k}}=0, \quad k=0,1,2,3, \quad 0<x<a, \quad \text { где } R_{a, T}=\{x, t: 0<x<a, 0<t<T\} .
\end{gathered}
$$

Решение задачи (2) получено в виде

$$
u(x, t)=2 a^{-1} \sum_{h=1}^{\infty} u^{h}(t) \sin \lambda_{h} x, \quad \lambda_{h}=\frac{h \pi}{a} .
$$

Доказано, что ряд (3) и ряды, полученные его дифференцированием $q$ раз по $t$ и $p$ раз по $x$, сходятся абсолютно и равномерно в $R_{a, T}$ при $p+q \leqslant 3$ и сходятся в $L^{2}\left(R_{a, T}\right)$ при $p+q=4$. Получены оценки для сумм этих рядов. Рассматривается также вспомогательная задача Коши: найти функцию $v(x, t)$ такую, что $v \in C^{3}\left(\overline{D_{\alpha, \beta}}\right) \cap H^{4}\left(D_{\alpha, \beta}\right)$,

$$
\Gamma v=f(x, t) \quad \text { в } D_{\alpha, \beta}, \quad \frac{\partial^{k} v}{\partial t^{k}}(x, 0)=0, \quad k=0,1,2,3, \quad \alpha \leqslant x \leqslant \beta,
$$

где $D_{\alpha, \beta}$ - характеристический треугольник с основанием $\alpha \leqslant x \leqslant \beta, \alpha, \beta=$ const. Задача Коши (4) сводится к задаче Коши для системы уравнений для вектор-функции $w=\left(w_{1}, w_{2}, w_{3}, w_{4}\right)$ вида

$$
\begin{gathered}
\frac{\partial w_{1}}{\partial x}-\frac{1}{2} \nu \frac{\partial w_{3}}{\partial x}-\frac{1}{2} \nu \frac{\partial w_{2}}{\partial t}+t_{0} \frac{\partial w_{4}}{\partial t}=(1+\varepsilon) w_{2}-w_{4}+f(x, t), \\
\frac{\partial w_{2}}{\partial x}-\frac{\partial w_{1}}{\partial t}=0, \quad \frac{\partial w_{3}}{\partial x}-\frac{\partial w_{2}}{\partial t}=0, \quad \frac{\partial w_{4}}{\partial x}-\frac{\partial w_{3}}{\partial t}=0, \quad \nu=1+t_{0}+\varepsilon t^{0} .
\end{gathered}
$$

Эта гиперболическая система приводится к каноническому виду. Решение задачи Коши получено методом последовательных приближений и даны оценки решения в соответствующих нормах. Далее, использяя совпадения решений задачи (2) и (4) в $R_{a, T} \cap D_{0, a}$, получена

Эта заметка подготовлена к опубликованию О. А. Олейник на основе незаконченной рукописи Г. Фикера. Он скончался 1 июня 1996 г. 
Tеорема 1. Решение задачи (1) в классе $C^{3}(\bar{Q}) \cap H^{4}(E)$ существует, единственно и в соответств ующ, их нормах непрерывно зависит от функиии $f$.

Построены приближенные решения задачи (1) и даны оценки сверху погрешности этих приближений. Рассматривается поведение решения задачи (1) при $t \rightarrow \infty$. При этом используется преобразование Лапласа. Введем дополнителшные условия:

1) $H_{1}$-существуют интегралы

$$
\int_{0}^{+\infty}\left|\frac{\partial f(x, t)}{\partial x}\right| d x, \quad \int_{0}^{+\infty}\left|\frac{\partial^{2} f(x, t)}{\partial x \partial t}\right| d x
$$

которые, как функции $t$, ограничены при $0<t<\infty$;

2) $H_{2}$-функция $f(x, t)$ - интегрируема в $Q$;

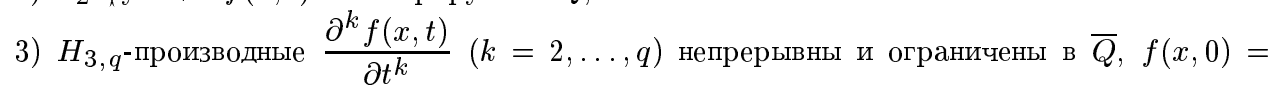
$\frac{\partial f(x, 0)}{\partial t}=\cdots=\frac{\partial^{k-2} f(x, 0)}{\partial t^{k-2}}=0$ для $x>0$.

ТеОрема 2. При условиях $H_{0}, H_{1}, H_{2}$ для решения $\varphi(x, t)$ задачи (1) выполнень соотношения:

$$
\begin{gathered}
\lim _{t \rightarrow \infty} \varphi(x, t) t^{-1}=0, \quad \lim _{t \rightarrow \infty} \frac{\partial \varphi}{\partial x}(x, t) t^{-\sigma}=0 \quad \text { для любого } \quad \sigma>0, \\
\lim _{t \rightarrow \infty} \frac{\partial \varphi}{\partial t}(x, t)(\log t)^{-1}=0, \quad \lim _{t \rightarrow \infty} \frac{\partial^{p+q} \varphi}{\partial x^{p} \partial t^{q}}(x, t)=0 \quad \text { для } \quad p+q=2,
\end{gathered}
$$

при условиях $H_{0}, H_{1}, H_{2}, H_{3,2}$ для любого $x>0$ и $p+q=3$, а при условиях $H_{0}, H_{1}, H_{2}$, $\mathrm{H}_{3,3}$ ир $+q=4$ имеем

$$
\lim _{t \rightarrow \infty} \frac{\partial^{p+q} \varphi(x, t)}{\partial x^{p} \partial t^{q}}=0 .
$$

\section{СПИСОК ЛИТЕРАТУРЫ}

[1] Hetnarski R. B., Ignaczak J. Generalized Thermoelasticity: Response of Semispace to a short Laser Pulse // Journal Thermal Stresses. 1994. V. 17. P. 377-396. 